\title{
Comment on 'Stage-dependent alterations of the serum cytokine pattern in colorectal carcinoma'
}

\author{
G Guthrie*,1 and D C McMillan ${ }^{1}$ \\ ${ }^{1}$ Academic Department of Surgery, School of Medicine, University of Glasgow, Glasgow Royal Infirmary, Glasgow \\ G31 2ER, UK
}

Sir,

We read with interest the work of Kantola and coworkers who examined the patterns of alterations of serum cytokine concentrations between normal controls and 148 patients newly diagnosed with colorectal cancer. Although in the main body of the paper they demonstrated clearly that the presence of colorectal cancer was associated with extensive alterations in the serum cytokine environment, it was also of interest that, within the colorectal cancer group, there were important findings to which the authors made little comment.

In particular, the data given within Tables 3 and 4 and Supplementary Tables 4, 5, 6, 7, 8, and 9 give a unique insight into the relationship between the serum cytokine alterations and the tumour-host systemic inflammatory response in patients with colorectal cancer. From these Tables, it is clear that sex, tumour site, and $\mathrm{N}$-stage had little association with the serum cytokine alterations reported. Furthermore, increasing T-stage was only associated with a significant increase in IL-6, IL-8, and MCP-1; increasing grade was only associated with a significant increase in IL-6 and IL-8; while the presence of metastasis was associated with a significant increase in IL-4, IL-6, IL-7, IL-8, MCP-1, and PDGF$\mathrm{BB}$. In contrast, the presence of a systemic inflammatory response, as evidenced by the modified Glasgow Prognostic Score (mGPS), was significantly associated with IL-1ra, IL-6, IL-7, IL-8, IL-9, IL-12, IFN- $\gamma$, IP-10, MCP-1, MIP-1B, and PDGF-BB. Taken together, these results would suggest that, in terms of influencing serum cytokine alterations, tumour-based factors have less effect than the activation of the systemic inflammatory response. Indeed, this is consistent with the report that the majority of patients do not resolve their systemic inflammatory response, following potentially curative surgery (McMillan et al, 2003; Guthrie et al, 2013) and the established prognostic value of the systemic inflammatory response in patients with colorectal cancer, before surgery (Roxburgh and McMillan, 2010).

Another interesting aspect of the work of Kantola and colleagues is that the association between mGPS and the serum cytokine alterations provides new insight into the inflammatory cells associated with the upregulation of the systemic inflammatory response in patients with colorectal cancer. With the exception of macrophages, few inflammatory cells can produce such a spectrum of cytokines and growth factors, and this is consistent with recent reports that macrophages are abundant in tumour microenvironments even in the absence of other inflammatory cells (Mohammed et al, 2012); (Richards et al, 2012a). Interestingly, the above findings are consistent with the hypothesis that tumour necrosis (increases with $\mathrm{T}$ stage) has an important role in linking local and systemic inflammatory responses in patients with colorectal cancer (Richards et al, 2012b).

It has long been recognised that tumour-associated macrophages localise to hypoxic regions of the tumour microenvironment. Therefore, it is plausible that the relative density of macrophages (perhaps M2) are important in such cytokine alterations and the elaboration of the systemic inflammatory response in patients with colorectal cancer. Alternatively, recent work has suggested that an accumulation of myeloid-derived suppressor cells in the tumour microenvironment may be important in these observations. Irrespectively, the above comments should encourage the authors to extend their studies in this area.

\section{REFERENCES}

Guthrie GJK, Roxburgh CSD, Horgan PG, McMillan DC (2013) Does interleukin-6 link explain the link between tumour necrosis, local and systemic inflammatory responses and outcome in patients with colorectal cancer? Cancer Treat Rev 39: 89-96.

McMillan DC, Canna K, Mcardle CS (2003) Systemic inflammatory response predicts survival following curative resection of colorectal cancer. Br J Surg 90: $215-219$.

Mohammed ZM, Going JJ, Edwards J, Elsberger B, Doughty JC, McMillan DC (2012) The relationship between components of tumour inflammatory cell 
infiltrate and clinicopathological factors and survival in patients with primary operable invasive ductal breast cancer. Br J Cancer 107: 864-873. Richards CH, Flegg KM, Roxburgh CSD, Going JJ, Mohammed Z, Horgan PG, McMillan DC (2012a) The relationships between cellular components of the peritumoural inflammatory response, clinicopathological characteristics and survival in patients with primary operable colorectal cancer. Br J Cancer 106: 2010-2015.
Richards CH, Roxburgh CS, Anderson JH, Mckee RF, Foulis AK, Horgan PG, McMillan DC (2012b) Prognostic value of tumour necrosis and host inflammatory responses in colorectal cancer. Br J Surg 99: 287-294.

Roxburgh CS, McMillan DC (2010) Role of systemic inflammatory response in predicting survival in patients with primary operable cancer. Future Oncol 6: 149-163. 